\title{
The stochastic nature of predator-prey cycles
}

\author{
Tânia Tomé ${ }^{\mathrm{a}, *}$, Áttila L. Rodrigues ${ }^{\mathrm{a}}$, Everaldo Arashiro ${ }^{\mathrm{a}, \mathrm{b}}$, Mário J. de Oliveira ${ }^{\mathrm{a}}$ \\ a Instituto de Física, Universidade de São Paulo, Caixa Postal 66318, 05314-970 São Paulo, SP, Brazil \\ ${ }^{\mathrm{b}}$ Departamento de Física, ICEB, Campus Morro do Cruzeiro, Universidade Federal de Ouro Preto, 35400-000 Ouro Preto, Minas Gerais, Brazil
}

\section{A R T I C L E I N F O}

Article history:

Received 30 September 2008

Received in revised form 18 December 2008

Accepted 8 January 2009

Available online 13 January 2009

\section{PACS:}

87.23.Cc

87.10.Hk

05.70.Ln

Keywords:

Population dynamics

Population cycles

Predator-prey systems

\begin{abstract}
A B S T R A C T
We study by numerical simulations the time correlation function of a stochastic lattice model describing the dynamics of coexistence of two interacting biological species that present time cycles in the number of species individuals. Its asymptotic behavior is shown to decrease in time as a sinusoidal exponential function from which we extract the dominant eigenvalue of the evolution operator related to the stochastic dynamics showing that it is complex with the imaginary part being the frequency of the population cycles. The transition from the oscillatory to the nonoscillatory behavior occurs when the asymptotic behavior of the time correlation function becomes a pure exponential, that is, when the real part of the complex eigenvalue equals a real eigenvalue. We also show that the amplitude of the undamped oscillations increases with the square root of the area of the habitat as ordinary random fluctuations.
\end{abstract}

(C) 2009 Elsevier B.V. All rights reserved.

\section{Introduction}

The coexistence of interacting biological species in their habitat is affected by random fluctuations [1,2]. The number of species individuals in a given territory fluctuates in time and must be regarded as stochastic noise. In an interacting biological species system that shows cycles in the number of species [3-5], the cycles are also affected by random fluctuations $[1,2]$ and must also be regarded as stochastic noise. In ordinary non-cyclic coexistence, the fluctuations are found to be ordinary Brownian noise, characterized by the exponential decay of the time correlation function. In cyclic coexistence, known as phase-forgetting quasi-cycles [1,2], however, the fluctuations are not ordinary Brownian noise but are characterized by exponentially decaying oscillations of the time correlation function.

The main purpose of this paper is to show, by studying a stochastic lattice model for predator-prey system, that the time behavior of species coexistence, is related to the dominant eigenvector of the evolution operator associated to the stochastic dynamics of population. When the corresponding eigenvalue is real, the decay of the time correlation function is exponential, the noise is ordinary Brownian, and the system shows ordinary species coexistence. When it is complex, the time correlation function is a sinusoidal exponential function, the noise is called chromatic

\footnotetext{
* Corresponding author.

E-mail address: ttome@if.usp.br (T. Tomé).
}

Brownian, and the system presents oscillatory species coexistence; the imaginary part being the frequency of the cycles.

We aim also to characterize the transition from an oscillatory coexistence of species to ordinary coexistence. A transition from one behavior to the other is defined as the point where the real part of the complex eigenvalue equals the real eigenvalue. The imaginary part of the complex eigenvalue, the frequency of oscillations, may act as an order parameter. In the present study we find that it jumps from a nonzero to a zero value characterizing a discontinuous transition. We remark however that this transition is not a phase transition in the thermodynamic sense, but is a real change in the behavior of biological population, that can actually be observed.

The above scenario will be revealed by the use of numerical simulations of the stochastic lattice model describing a predatorprey system, introduced in 1994 by Satulovsky and Tomé (ST) [6], which displays stable local self-sustained oscillations called also endogenous quasi-cycles [1]. This model takes into account two essential features in describing population dynamics: the stochasticity embodied in a biological population [1,2] and the spatial structure of the habitat $[7,8]$ and belongs to a class of stochastic lattice models with stochastic local rules [9-22]. Models in this class are continuous Markov processes defined by discrete stochastic variables residing on the sites of the lattice, that is, governed by a master equation, incorporating the discreteness of the species individuals and local interactions.

A remarkable property of the stochastic lattice model studied here is that the amplitude of the undamped cycles in the 
number of species individuals in a given territory increases with the square root of the area of the territory [11,23-25], the same property valid for ordinary stochastic fluctuations. The undamped oscillations are intertwined with the stochastic fluctuations and are not expected to be synchronized oscillations, whose amplitude would increase with the area and should be identified as phase-remembering quasi-cycles [1,2]. The unsynchronized cyclic fluctuations, or phase-forgetting quasi-cycles, nevertheless, survive at a local level but cannot be distinguished from the ordinary fluctuations by a static property such as the amplitude. They should be characterized by a dynamic property such as the time correlation function which will be determined here by numerical simulations.

\section{Model}

We consider here the stochastic ST lattice model [6] for a predator-prey system. Each site of a regular square lattice can be either empty or occupied by one individual of different species. The state of a site is described by a variable $\eta_{i}$ that takes the values 0,1 , or 2 , according to whether the site is empty, occupied by a prey individual or by a predator, respectively. For convenience we define the indicators $p_{i}, n_{i}$, and $m_{i}$ as variables that take the value 1 if the site $i$ is empty, occupied by a prey individual and by a predator, respectively, and zero otherwise.

The model is composed by three processes that are described as follows: (a) $(0 \rightarrow 1)$ prey individuals are born in empty sites; (b) $(1 \rightarrow 2)$ prey individual dies and is instantaneously replaced by a new born predator; (c) $(2 \rightarrow 0)$ a predator can die leaving an empty site. The two first processes are catalytic whereas the third is spontaneous. The transition rate $w_{i}(\eta)$ from a configuration $\eta=\left\{\eta_{j}\right\}$ to another configuration such that the state of site $i$ is changed in the cyclic order $0 \rightarrow 1 \rightarrow 2 \rightarrow 0$ is given by

$w_{i}(\eta)=p_{i} \frac{a}{4} \sum_{\delta} n_{i+\delta}+n_{i} \frac{b}{4} \sum_{\delta} m_{i+\delta}+c m_{i}$,

where the summation is over the nearest neighbor sites and $a, b$ and $c$ are three parameters related to the three processes.

The probability $P(\eta, t)$ of configuration $\eta$ at time $t$ evolves in time according to the Master equation

$\frac{d}{d t} P(\eta, t)=\mathcal{W} P(\eta, t)$,

where $\mathcal{W}$ is the operator defined by

$\mathcal{W} \phi(\eta)=\sum_{i}\left\{w_{i}\left(\eta^{i}\right) P\left(\eta^{i}, t\right)-w(\eta) \phi(\eta)\right\}$,

where the state denoted by $\eta^{i}$ is obtained from $\eta$ by a anticyclic permutation of the state of site $i$, that is, $2 \rightarrow 1 \rightarrow 0 \rightarrow 2$.

As one varies the three parameter $a, b$ and $c$, one finds an active stationary state, that can be of two types, with and without oscillations, and for sufficient large values of $c$ an absorbing state with prey repletion [6]. By rescaling time it is always possible to assume that $a+b+c=1$, with $0 \leqslant a, b, c \leqslant 1$. Indeed, suppose that we are given values of parameter $a^{\prime}, b^{\prime}$ and $c^{\prime}$ such that their sum $s$ is distinct from unity. If we divide both members of the master equation (3) by $s$ and rescale time $t^{\prime}=s t$ then the new parameters will be $a=a^{\prime} / s, b=b^{\prime} / s$ and $c=c^{\prime} / s$ which sum up to 1 .

We have simulated the ST model on a square lattice of $N=$ $L \times L$ sites, which is to be regarded as proportional to the area of the territory where the species survive, with periodic boundary conditions, for $a=b=(1-c) / 2$ for several values of $c$. For small values of $c$, the model displays species coexistence in which the density of predator is nonzero and the density of prey is strictly less than one, called an active state. Increasing $c$, the density of

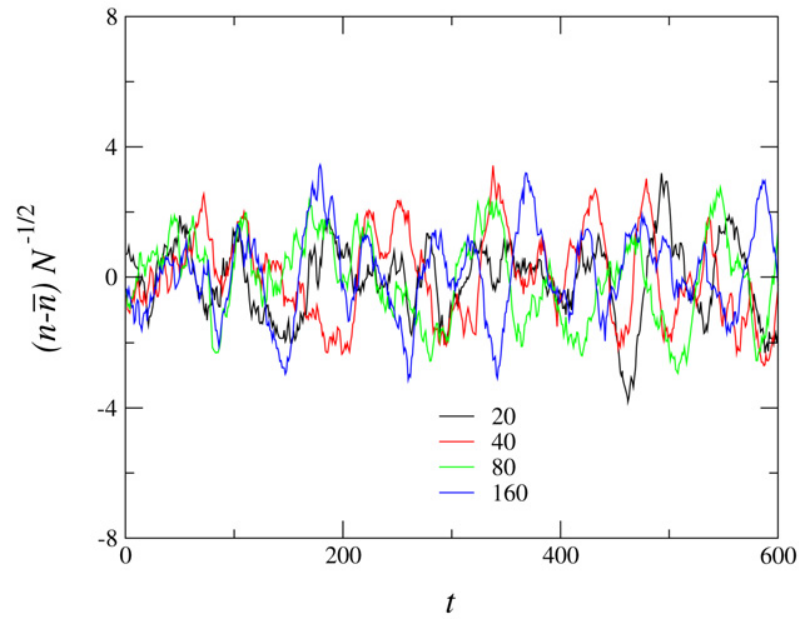

Fig. 1. Deviation $n-\bar{n}$ in the number of prey individuals as a function of time $t$, showing noisy oscillations that scales with $\sqrt{N}$, from numerical simulations on a square lattice of $N=L \times L$ sites, for several values of $L$, for the case $a=b$ and $c=0.10$.

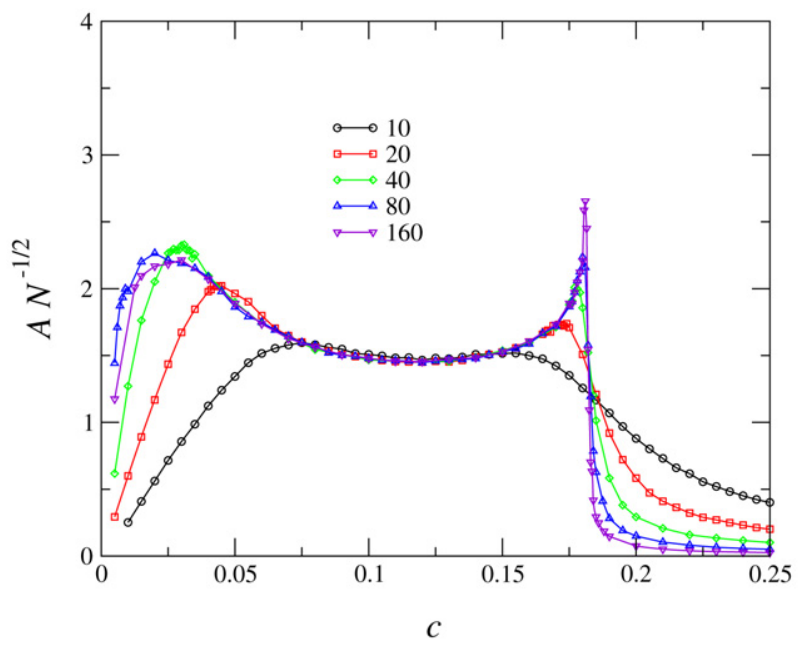

Fig. 2. Amplitude of fluctuations in the number of prey $A=\sqrt{\left\langle n^{2}\right\rangle-\bar{n}^{2}}$ as a function of $c$ for several values of the system sizes $L$, for $a=b$, obtained from numerical simulations on a square lattice of $N=L \times L$ sites.

predator vanishes at a critical point $c=c_{c}$, the density of prey becomes one, and the lattice becomes full of prey, characterizing an absorbing state. Our numerical simulations gives $c_{c}=0.182(1)$.

The deviation $n-\bar{n}$ of the number of prey $n$ from its average $\bar{n}=\langle n\rangle$ scales as $n-\bar{n} \sim \sqrt{N}$ as can be seen in Fig. 1 for $c=$ 0.10 , where oscillatory noise can be observed. To check this scaling we determined the mean amplitude $A$, defined by $A=\sqrt{\left\langle n^{2}\right\rangle-\bar{n}^{2}}$. From Fig. 2 it follows that indeed the amplitude scales as $A \sim \sqrt{N}$, for sufficiently high values of $N$.

\section{Time correlation}

In order to calculate time correlation functions, we need to determine the conditional probability $K\left(\eta, t \mid \eta^{\prime}, t^{\prime}\right)$ of configuration $\eta$ at time $t$ giving the configuration $\eta^{\prime}$ at a previous time $t^{\prime}$. This quantity obeys the Chapman-Kolmogorov equation which in differential form is given by

$\frac{d}{d t} K\left(\eta, t \mid \eta^{\prime}, t^{\prime}\right)=\mathcal{W} K\left(\eta, t \mid \eta^{\prime}, t^{\prime}\right)$,

with the initial condition $K\left(\eta, t^{\prime} ; \eta^{\prime}, t^{\prime}\right)=\delta\left(\eta, \eta^{\prime}\right)$. 


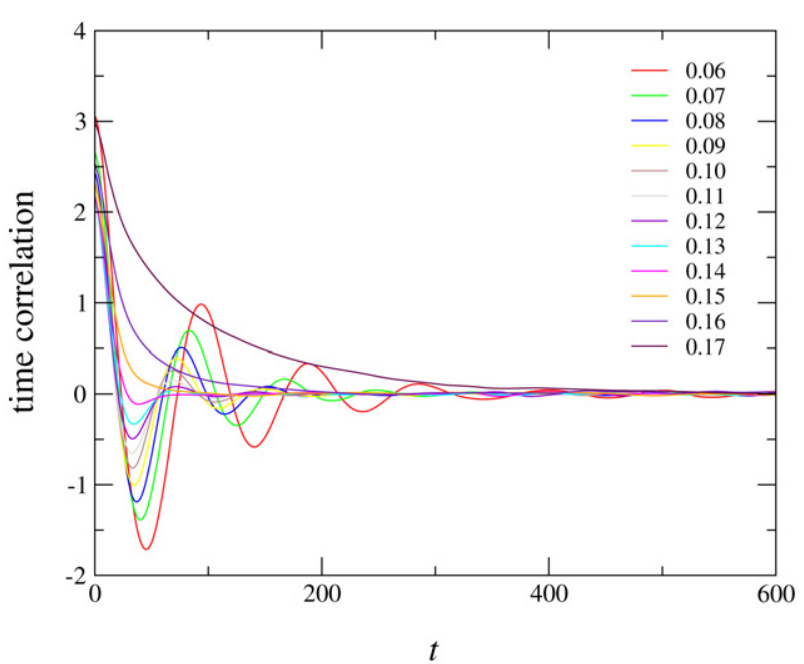

Fig. 3. Prey-prey time autocorrelation function versus the time lag $t$, determined by numerical simulations on a square lattice with $N=100 \times 100$ sites for several values of $c$ for the case $a=b$.

A prey-prey autocorrelation function in the stationary regime is defined by

$c_{1}(t)=\sum_{\eta} \sum_{\eta^{\prime}} n_{i} K\left(\eta, t \mid \eta^{\prime}, 0\right) x^{\prime} P\left(\eta^{\prime}\right)-\bar{x}^{2}$,

where $x^{\prime}=n^{\prime} / N$ and $n^{\prime}$ is the number of prey individuals in configuration $\eta^{\prime}$, and $\bar{x}=\langle x\rangle$. Other time correlations can be defined by similar expressions.

Numerically, we have used the following expression for the prey autocorrelation function: $c_{1}(t+s, s)=\langle(x(t+s)-\bar{x})(x(s)-\bar{x})\rangle$ where $t$ is the time lag. The quantity $s$ is considered to be large enough for the system to be in the stationary regime, occurring after the transient. Fig. 3 shows the time correlation function as a function of the time lag for several values of $c$ inside the species coexistence interval. In all cases the correlation decreases with the time lag $t$, vanishing when $t \rightarrow \infty$. Two regimes can be distinguished. One with oscillations and the other without.

For large values of $t$, the conditional probability $K$ approaches the stationary probability distribution. Asymptotically, we have

$K\left(\eta, t \mid \eta^{\prime}, 0\right)=P(\eta)+\Phi(\eta) X\left(\eta^{\prime}\right) e^{\lambda t}$,

where $\lambda$ is the eigenvalue with the greatest real part, which we call dominant eigenvalue, of the operator $\mathcal{W}$ and $\Phi$ and $X$ their corresponding right and left eigenfunctions. Substitution of this expression into Eq. (5) gives the following asymptotic behavior for the prey-prey time autocorrelation

$c_{1}(t)=B e^{\lambda t}$.

When $\lambda$ is complex with a nonzero imaginary part, that is, $\lambda=$ $-\alpha \pm i \omega$, the time correlation function is

$c_{1}(t)=B_{1} e^{-\alpha t} \cos (\omega t)$,

which describes a chromatic Brownian noise. If the dominant eigenvalue is real, $\lambda=-\gamma$, the time correlation is an exponential

$c_{1}(t)=B_{2} e^{-\gamma t}$,

which describes an ordinary Brownian noise. We have determined for each correlation function shown in Fig. 3 the values of $\alpha, \omega$ and $\gamma$ by fitting to the data points the right-hand side of either Eqs. (8) or (9). When $\alpha \approx \gamma$ we have used instead the function

$c_{1}(t)=B_{1} e^{-\alpha t} \cos (\omega t)+B_{2} e^{-\gamma t}$,

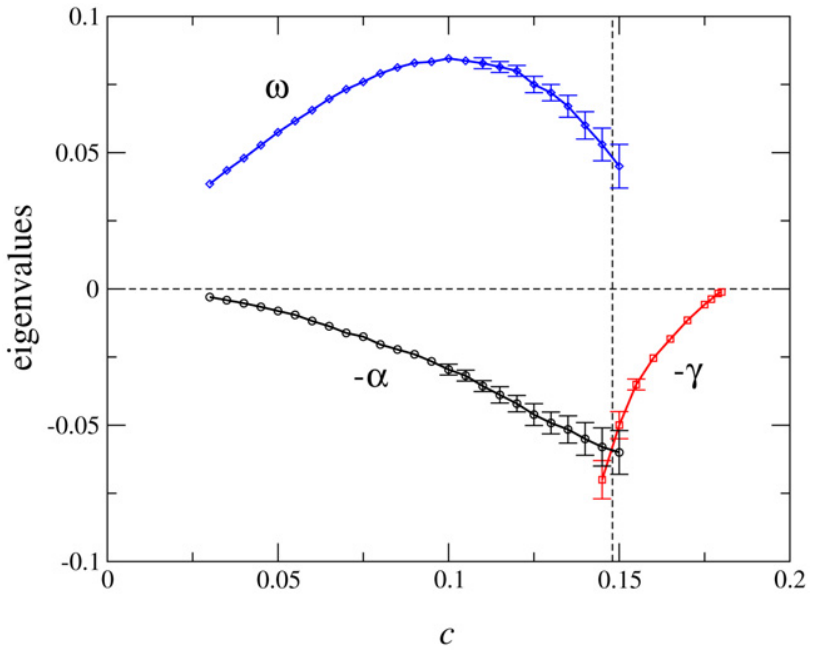

Fig. 4. Real eigenvalue $(-\gamma)$, real $(-\alpha)$ and imaginary $(\omega)$ parts of the complex eigenvalue as determined from numerical simulations. The vertical dotted line at $c^{*}=0.148$ indicates the transition from oscillatory to ordinary species coexistence. The phase transition from active to absorbing state occurs at $c_{c}=0.182$ where $\gamma \rightarrow 0$.

which is the sum of the right-hand sides of Eqs. (8) and (9). The three quantities are plotted as a function of $c$, as shown in Fig. 4. The change in behavior happens when $\alpha=\gamma$ which occurs at $c^{*}=0.148(4)$. Notice that $\omega \rightarrow \omega_{0}=0.057(9)$, a finite value, when $c \rightarrow c^{*}$

\section{Conclusion}

In summary, we have shown by numerical simulation that the phase-forgetting quasi-cycles in stochastic lattice models are related to the complex "gap" of the evolution operator; the imaginary part of the "gap" being identified as the frequency of oscillations. We remark that the emergence of a complex eigenvalue, leading to a sinusoidal exponential decaying of the time correlation function, observed in real population data [1], is only possible because the dynamics lacks detailed balance (is microscopically irreversible). The evolution operator of a system that obeys detailed balance (microscopically reversible) exhibits solely real eigenvalues. The amplitude of the noisy oscillations on the number of species individuals increases with $\sqrt{N}$, in the same way as ordinary stochastic fluctuations, characterizing unsynchronized oscillations.

\section{References}

[1] R.M. Nisbet, W.S.C. Gurney, Modelling Fluctuating Populations, Wiley, New York, 1982.

[2] E. Renshaw, Modelling Biological Populations in Space and Time, Cambridge University Press, Cambridge, 1991.

[3] S. Utida, Ecology 38 (1957) 442.

[4] B.E. Kendall, C.J. Briggs, W.W. Murdoch, P. Turchin, S.P. Ellner, E. McCauley, R.M. Nisbet, S.N. Wood, Ecology 80 (1999) 1789

[5] M. Pineda-Krch, H.J. Blok, U. Dieckmann, M. Doebeli, Oikos 116 (2007) 53.

[6] J. Satulovsky, T. Tomé, Phys. Rev. E 49 (1994) 5073.

[7] D. Tilman, P. Kareiva, Spatial Ecology: the Role of Space in Population Dynamics and Interactions, Princeton University Press, Princeton, 1997.

[8] R. Durrett, S. Levin, Theor. Popul. Biol. 46 (1994) 363.

[9] K. Tainaka, J. Phys. Soc. Jpn. 57 (1988) 2588.

[10] L. Fracheburg, P. Krapvisky, J. Phys. A 31 (1998) L287.

[11] A. Lipowski, Phys. Rev. E 60 (1999) 5179.

[12] A. Provata, G. Nicolis, F. Baras, J. Chem. Phys. 110 (1999) 8361.

[13] M. Droz, A. Pękalski, Phys. Rev. E 63 (2001) 051909.

[14] T. Antal, M. Droz, A. Lipowsky, G. Odor, Phys. Rev. E 64 (2001) 036118.

[15] M.A.M. de Aguiar, E.M. Rauch, Y. Bar-Yam, Phys. Rev. E 67 (2003) 047102.

[16] G. Szabó, J. Phys. A 38 (2005) 6689. 
[17] D. Stauffer, A. Kunwar, D. Chowdhury, Physica A 352 (2005) 202.

[18] K.C. de Carvalho, T. Tomé, Int. Mod. Phys. C 17 (2006) 1647.

[19] M. Mobilia, I.T. Georgiev, U.C. Täuber, Phys. Rev. E 73 (2006) 040903.

[20] E. Arashiro, T. Tomé, J. Phys. A 40 (2007) 887.

[21] A.L. Rodrigues, T. Tomé, Braz. J. Phys. 38 (2008) 87.
[22] E. Arashiro, A.L. Rodrigues, M.J. de Oliveira, T. Tomé, Phys. Rev. E 77 (2008) 061909.

[23] T. Antal, M. Droz, Phys. Rev. E 63 (2001) 056119.

[24] A.J. McKane, T.J. Newman, Phys. Rev. Lett. 94 (2005) 218102.

[25] S. Morita, K. Tainaka, Popul. Ecol. 48 (2006) 99. 